\title{
SOLVING METHOD FOR STABILITY PROBLEM OF ELASTOPLASTIC CYLINDRICAL SHELLS WITH COMPRESSIBLE MATERIAL SUBJECTED TO COMPLEX LOADING PROCESSES
}

\author{
DAO VAN DUNG \\ Vietnam National University, Hanoi
}

\begin{abstract}
The system of stability equations of elasto plastic cylindrical shell made of compressible material was established in work [3]. In the present paper, we study the solution of the problems and methods for determining the critical load. The obtained results describe the influence of the compressibility of material on the stability of the shell. When a material is incompressible, these results reduce to the previous well-known ones (see $[1,2$, $4,5])$.
\end{abstract}

\section{Stability problem}

Let's consider a cylindrical shell of length $L$, radius $R$ and thickness $h$. We choose $x_{1}$ lying along the generatrix of the shell, $x_{2}=R \theta_{1}$ with $\theta_{1}$ - the angle of circular arc and $z$ in the direction of the normal to the middle surface.

Assume that a material is compressible. We consider the shell being acted upon by the external forces $p_{11}, p_{12}, p_{22}$ which depend arbitrarily on a loading parameter $t$. One of the main aims of the stability problem is to find the moment $t_{*}$ when the instability of structure happens and respectively the critical loads, $p_{i j}^{*}=p_{i j}\left(t_{*}\right)$. Suppose that the unloading does not happen in the structure. We use the criterion of bifurcation of equilibrium state to study the proposed problem.

An analysis of the elastoplastic stability problem is always made in two parts: pre-buckling process and post-buckling process.

\subsection{Pre-buckling process}

Suppose that at any moment, $t$ there exists a membrane plane stress state in the shell

$$
\sigma_{11}=-p_{11}, \sigma_{12}=-p_{12}, \sigma_{22}=-p_{22}, \sigma_{13}=\sigma_{23}=\sigma_{33}=0
$$

So that

$$
\begin{aligned}
\sigma & =\frac{\sigma_{11}+\sigma_{22}}{3}=-\frac{p_{11}+p_{22}}{3} \\
\sigma_{u} & =\sqrt{\sigma_{11}^{2}-\sigma_{11} \sigma_{22}+\sigma_{22}^{2}+3 \sigma_{12}^{2}}=\sqrt{p_{11}^{2}-p_{11} p_{22}+p_{22}^{2}+3 p_{12}^{2}}
\end{aligned}
$$


Components of the strain velocity tensor determined according to the theory of elastoplastic processes [1] are of the form

$$
\begin{aligned}
& \dot{\varepsilon}_{11}=\frac{1}{N}\left(-\dot{p}_{11}+\frac{1}{2} \dot{p}_{22}\right)-Q(s, t)\left(p_{11}-\frac{1}{2} p_{22}\right)-\frac{1}{9 K}\left(\dot{p}_{11}+\dot{p}_{22}\right), \\
& \dot{\varepsilon}_{22}=\frac{1}{N}\left(-\dot{p}_{22}+\frac{1}{2} \dot{p}_{11}\right)-Q(s, t)\left(p_{22}-\frac{1}{2} p_{11}\right)-\frac{1}{9 K}\left(\dot{p}_{11}+\dot{p}_{22}\right), \\
& \dot{\varepsilon}_{33}=\frac{1}{2 N}\left(\dot{p}_{11}+\dot{p}_{22}\right)+\frac{1}{2} Q(s, t)\left(p_{11}+p_{22}\right)-\frac{1}{9 K}\left(\dot{p}_{11}+\dot{p}_{22}\right), \\
& \dot{\varepsilon}_{12}=-\frac{3 \dot{p}_{12}}{2 N}-\frac{3}{2} Q(s, t) p_{12} .
\end{aligned}
$$

where

$$
\begin{aligned}
Q(s, t) & =\left(\frac{1}{\phi^{\prime}}-\frac{1}{N}\right) \frac{1}{\sigma_{u}^{2}}\left(p_{11} \dot{p}_{11}+p_{22} \dot{p}_{22}-\frac{1}{2} p_{11} \dot{p}_{22}-\frac{1}{2} p_{22} \dot{p}_{11}+3 p_{12} \dot{p}_{12}\right), \\
\phi^{\prime} & =\phi^{\prime}(s), \quad N=\frac{\sigma_{u}}{s} .
\end{aligned}
$$

The arc-length of the strain trajectory is given respectively by the formula

$$
\frac{d s}{d t}=\frac{\sqrt{2}}{3}\left[\left(\dot{\varepsilon}_{11}-\dot{\varepsilon}_{22}\right)^{2}+\left(\dot{\varepsilon}_{22}-\dot{\varepsilon}_{33}\right)^{2}+\left(\dot{\varepsilon}_{33}-\dot{\varepsilon}_{11}\right)^{2}+6 \dot{\varepsilon}_{12}^{2}\right]^{1 / 2} \equiv F(s, t) .
$$

So, we can determine, from obtained equations, stress and strain states of the cylindrical shell in the pre-buckling process.

\subsection{Post - buckling process}

As shown in [3], the system of stability equations of the cylindrical shell is written in the form

$$
\begin{aligned}
& \beta_{1} \frac{\partial^{4} \varphi}{\partial x_{1}^{4}}+\beta_{2} \frac{\partial^{4} \varphi}{\partial x_{1}^{3} \partial x_{2}}+\beta_{3} \frac{\partial^{4} \varphi}{\partial x_{1}^{2} \partial x_{2}^{2}}+\beta_{4} \frac{\partial^{4} \varphi}{\partial x_{1} \partial x_{2}^{3}} \\
& \quad+\beta_{5} \frac{\partial^{4} \varphi}{\partial x_{2}^{4}}+\frac{N}{R} \frac{\partial^{2} \delta w}{\partial x_{1}^{2}}=0, \\
& \alpha_{1} \frac{\partial^{4} \delta w}{\partial x_{1}^{4}}+\alpha_{2} \frac{\partial^{4} \delta w}{\partial x_{1}^{3} \partial x_{2}}+\alpha_{3} \frac{\partial^{4} \delta w}{\partial x_{1}^{2} \partial x_{2}^{2}}+\alpha_{4} \frac{\partial^{4} \delta w}{\partial x_{1} \partial x_{2}^{3}}+\alpha_{5} \frac{\partial^{4} \delta w}{\partial x_{2}^{4}} \\
& \quad+\frac{9}{N h^{2}}\left(p_{11} \frac{\partial^{2} \delta w}{\partial x_{1}^{2}}+2 p_{12} \frac{\partial^{2} \delta w}{\partial x_{1} \partial x_{2}}+p_{22} \frac{\partial^{2} \delta w}{\partial x_{2}^{2}}-\frac{1}{R} \frac{\partial^{2} \varphi}{\partial x_{1}^{2}}\right)=0 .
\end{aligned}
$$

where

$$
\begin{aligned}
& \beta_{1}=1+\frac{1}{4}\left(\frac{N}{\phi^{\prime}}-1\right)\left(\frac{2 \sigma_{22}-\sigma_{11}}{\sigma_{u}}\right)^{2}+\frac{N}{9 K}, \\
& \beta_{2}=-3\left(\frac{N}{\phi^{\prime}}-1\right) \frac{\sigma_{12}\left(2 \sigma_{22}-\sigma_{11}\right)}{\sigma_{u}^{2}},
\end{aligned}
$$




$$
\begin{aligned}
\beta_{3}= & 2\left(1+\frac{N}{9 K}\right)+\frac{1}{2}\left(\frac{N}{\phi^{\prime}}-1\right) \frac{\left(2 \sigma_{22}-\sigma_{11}\right)\left(2 \sigma_{11}-\sigma_{22}\right)}{\sigma_{u}^{2}}+9\left(\frac{N}{\phi^{\prime}}-1\right)\left(\frac{\sigma_{12}}{\sigma_{u}}\right)^{2}, \\
\beta_{4}= & -3\left(\frac{N}{\phi^{\prime}}-1\right) \frac{\sigma_{12}\left(2 \sigma_{11}-\sigma_{22}\right)}{\sigma_{u}^{2}}, \\
\beta_{5}= & 1+\frac{1}{4}\left(\frac{N}{\phi^{\prime}}-1\right)\left(\frac{2 \sigma_{11}-\sigma_{22}}{\sigma_{u}}\right)^{2}+\frac{N}{9 K} \\
\alpha_{1}= & \frac{3}{4 N}\left[\left(D_{11} \sigma_{11}-D_{12} \sigma_{22}\right) \frac{\sigma_{11}}{\sigma_{u}^{2}}+2 D_{13}-D_{14}\right] \\
\alpha_{2}= & \frac{3}{4 N}\left[2\left(D_{11} \sigma_{11}-D_{12} \sigma_{22}\right) \frac{\sigma_{12}}{\sigma_{u}^{2}}+2 D_{31} \frac{\sigma_{12} \sigma_{11}}{\sigma_{u}^{2}}-4 D_{32}-2 D_{33}\right] \\
\alpha_{3}= & \frac{3}{4 N}\left[\left(D_{11} \sigma_{11}-D_{12} \sigma_{22}\right) \frac{\sigma_{22}}{\sigma_{u}^{2}}+D_{13}-2 D_{14}+\left(-D_{21} \sigma_{11}+D_{22} \sigma_{22}\right) \frac{\sigma_{11}}{\sigma_{u}^{2}}\right. \\
& \left.-2 D_{23}+D_{24}+4 D_{31} \frac{\sigma_{12}^{2}}{\sigma_{u}^{2}}+2 D_{34}\right] \\
\alpha_{4}= & \frac{3}{4 N}\left[2\left(-D_{21} \sigma_{11}+D_{22} \sigma_{22}\right) \frac{\sigma_{12}}{\sigma_{u}^{2}}+2 D_{31} \frac{\sigma_{12} \sigma_{22}}{\sigma_{u}^{2}}-2 D_{32}-4 D_{33}\right] \\
\alpha_{5}= & \frac{3}{4 N}\left[\left(-D_{21} \sigma_{11}+D_{22} \sigma_{22}\right) \frac{\sigma_{22}}{\sigma_{u}^{2}}-D_{23}+2 D_{24}\right] .
\end{aligned}
$$

The coefficients $D_{i j}$ in (1.6) are calculated as follows [3]

$$
\begin{array}{rlrl}
D_{11}=\frac{A}{C}\left(1+\frac{B}{3 K}+A \frac{\sigma \sigma_{22}}{3 K \sigma_{u}^{2}}\right), & D_{12}=\frac{A}{C}\left(\frac{B}{3 K}+A \frac{\sigma \sigma_{11}}{3 K \sigma_{u}^{2}}\right), \\
D_{13}=\frac{B}{C}\left(1+\frac{B}{3 K}+A \frac{\sigma \sigma_{22}}{3 K \sigma_{u}^{2}}\right), & D_{14}=\frac{B}{C}\left(\frac{B}{3 K}+A \frac{\sigma \sigma_{11}}{3 K \sigma_{u}^{2}}\right), \\
D_{21}=\frac{A}{C}\left(\frac{B}{3 K}+A \frac{\sigma \sigma_{22}}{3 K \sigma_{u}^{2}}\right), & D_{22}=\frac{A}{C}\left(1+\frac{B}{3 K}+A \frac{\sigma \sigma_{11}}{3 K \sigma_{u}^{2}}\right), \\
D_{23}=\frac{B}{C}\left(\frac{B}{3 K}+A \frac{\sigma \sigma_{22}}{3 K \sigma_{u}^{2}}\right), & D_{24}=\frac{B}{C}\left(1+\frac{B}{3 K}+A \frac{\sigma \sigma_{11}}{3 K \sigma_{u}^{2}}\right), \\
D_{31}=A-\frac{A^{2}}{C} \cdot \frac{\sigma\left(\sigma_{11}+\sigma_{22}\right)}{3 K \sigma_{u}^{2}}, & D_{32}=\frac{A B}{C} \frac{\sigma \sigma_{12}}{3 K \sigma_{u}^{2}}, \quad D_{33}=\frac{A B}{C} \frac{\sigma \sigma_{12}}{3 K \sigma_{u}^{2}}, \\
D_{34}=B, \quad C=1+\frac{2 B}{3 K}+A \frac{\sigma\left(\sigma_{11}+\sigma_{22}\right)}{3 K \sigma_{u}^{2}}, \quad A=\phi^{\prime}-N, \quad B=\frac{2}{3} N .
\end{array}
$$

In order to solve the stability problem of shell, we suppose that the kinematic boundary condition is simply supported at the planes $x_{1}=0$ and $x_{1}=L$. 


\section{Method of solution}

We find the solution $\delta w$ in the form

$$
\delta w=A_{m n} \sin \left(\frac{m \pi x_{1}}{L}+\frac{n x_{2}}{R}\right) .
$$

It is easy to see that this solution satisfies the kinematic boundary condition in the sense of Saint-Venant.

Using the expression of $\delta w$ and the equation (1.4), we obtain a relation for determining the function $\varphi$. The particular solution of this equation is of the form

$$
\varphi=B_{m n} \sin \left(\frac{m \pi x_{1}}{L}+\frac{n x_{2}}{R}\right)
$$

where

$$
\begin{aligned}
B_{m n}=\frac{N}{R} A_{m n}[ & \beta_{1}\left(\frac{m \pi}{L}\right)^{2}+\beta_{2}\left(\frac{m \pi}{L}\right)\left(\frac{n}{R}\right)+\beta_{3}\left(\frac{n}{R}\right)^{2} \\
& \left.+\beta_{4}\left(\frac{L}{m \pi}\right)\left(\frac{n}{R}\right)^{3}+\beta_{5}\left(\frac{L}{m \pi}\right)^{2}\left(\frac{n}{R}\right)^{4}\right]^{-1}
\end{aligned}
$$

Substituting (2.1) and (2.2) into the stability equation (1.5) and taking into account the existence of non-trivial solution i.e.' $A_{m n} \neq 0$, we receive the expression for defining critical loads

$$
\begin{aligned}
& p_{11}\left(\frac{m \pi}{L}\right)^{2}+2 p_{12}\left(\frac{m \pi}{L}\right)\left(\frac{n}{R}\right)+p_{22}\left(\frac{n}{R}\right)^{2}= \\
& =N\left\{\frac { h ^ { 2 } } { 9 } \left[\alpha_{1}\left(\frac{m \pi}{L}\right)^{4}+\alpha_{2}\left(\frac{m \pi}{L}\right)^{3}\left(\frac{n}{R}\right)+\alpha_{3}\left(\frac{m \pi}{L}\right)^{2}\left(\frac{n}{R}\right)^{2}\right.\right. \\
& \left.\quad+\alpha_{4}\left(\frac{m \pi}{L}\right)\left(\frac{n}{R}\right)^{3}+\alpha_{5}\left(\frac{n}{R}\right)^{4}\right]+\left(\frac{m \pi}{L}\right)^{2}\left[\beta_{1}\left(\frac{m \pi R}{L}\right)^{2}\right. \\
& \left.\left.+\beta_{2}\left(\frac{m \pi R}{L}\right) n+\beta_{3} n^{2}+\beta_{4}\left(\frac{L}{m \pi R}\right) n^{3}+\beta_{5}\left(\frac{m}{m \pi R}\right)^{2} n^{4}\right]^{-1}\right\} .
\end{aligned}
$$

By putting $\psi=n^{2}, \beta=\frac{m \pi R}{n L}, i=\frac{3 R}{h}$ (called the slenderness of the shell), the relation (2.4) is written in the form

$$
\begin{aligned}
i^{2}=\frac{N}{p_{11} \beta^{2}+2 p_{12} \beta+p_{22}} & {\left[\left(\alpha_{1} \beta^{4}+\alpha_{2} \beta^{3}+\alpha_{3} \beta^{2}+\alpha_{4} \beta+\alpha_{5}\right) \psi\right.} \\
+ & \left.\frac{i^{2} \beta^{4}}{\left(\beta_{1} \beta^{4}+\beta_{2} \beta^{3}+\beta_{3} \beta^{2}+\beta_{4} \beta+\beta_{5}\right) \psi}\right] .
\end{aligned}
$$

From here, we get

$$
i^{2}=\frac{N \psi^{2}\left(\alpha_{1} \beta^{4}+\alpha_{2} \beta^{3}+\alpha_{3} \beta^{2}+\alpha_{4} \beta+\alpha_{5}\right)\left(\beta_{1} \beta^{4}+\beta_{2} \beta^{3}+\beta_{3} \beta^{2}+\beta_{4} \beta+\beta_{5}\right)}{\left(p_{11} \beta^{2}+2 p_{12} \beta+p_{22}\right)\left(\beta_{1} \beta^{4}+\beta_{2} \beta^{3}+\beta_{3} \beta^{2}+\beta_{4} \beta+\beta_{5}\right) \psi-N \beta^{4}} .
$$


Because of the complex loading process depending on a parameter $t$, the quantities in the expression (2.6) are functions of $t$ and $s$. Besides, the arc-length of the strain trajectory $s$ from (1.3) is a function of $t$, too. So, we have to solve simultaneously the equations (1.3) and (2.6) by the loading parameter method [1]. After determining the critical value $t_{*}$, we can find the critical loads as follows

$$
p_{11}^{*}=p_{11}\left(t_{*}\right), \quad p_{12}^{*}=p_{12}\left(t_{*}\right), \quad p_{22}^{*}=p_{22}\left(t_{*}\right) .
$$

Hereafter, we will consider some concrete problems.

\section{Some concrete problems}

\subsection{Cylindrical shell subjected to compression along the generatrix}

In this case, we get

$$
\begin{aligned}
& \sigma_{11}=-p, \quad \sigma_{12}=\sigma_{22}=0, \quad \sigma_{u}=\left|\sigma_{11}\right|=p, \\
& \dot{\varepsilon}_{11}=-\frac{\dot{p}}{\phi^{\prime}}-\frac{\dot{p}}{9 K}, \quad \dot{\varepsilon}_{22}=\frac{\dot{p}}{2 \phi^{\prime}}-\frac{\dot{p}}{9 K}, \quad \dot{\varepsilon}_{33}=\frac{\dot{p}}{2 \phi^{\prime}}-\frac{\dot{p}}{9 K}, \quad \dot{\varepsilon}_{12}=0, \\
& \frac{d s}{d t}=\frac{\dot{p}(t)}{\phi^{\prime}(s)} \quad \text { or } \quad s=\phi^{-1}\left(\sigma_{u}\right)=\phi^{-1}(p) .
\end{aligned}
$$

The coefficients in (1.6) and (1.7) are of the form

$$
\begin{array}{ll}
\alpha_{1}=\frac{1}{4 C}+\frac{3 \phi^{\prime}}{4 N C}+\frac{\phi^{\prime}}{9 K C}, & \alpha_{2}=\alpha_{4}=0, \\
\alpha_{3}=1+\frac{1}{C}-\frac{2 \phi^{\prime}}{9 K C}, & \alpha_{5}=\frac{1}{C}+\frac{\phi^{\prime}}{9 K C}, \\
\beta_{1}=\frac{3}{4}+\frac{N}{4 \phi^{\prime}}+\frac{N}{9 K}, & \beta_{2}=\beta_{4}=0, \\
\beta_{3}=3-\frac{N}{\phi^{\prime}}+\frac{2 N}{9 K}, & \beta_{5}=\frac{N}{\phi^{\prime}}+\frac{N}{9 K} \\
C=1+\frac{3 N+\phi^{\prime}}{9 K} . &
\end{array}
$$

The relation (2.6) becomes

$$
i^{2}=\frac{N \psi^{2}\left(\alpha_{1} \beta^{4}+\alpha_{3} \beta^{2}+\alpha_{5}\right)\left(\beta_{1} \beta^{4}+\beta_{3} \beta^{2}+\beta_{5}\right)}{p \psi \beta^{2}\left(\beta_{1} \beta^{4}+\beta_{3} \beta^{2}+\beta_{5}\right)-N \beta^{4}} .
$$

Putting $\theta=\beta^{2}=\left(\frac{m \pi R}{n L}\right)^{2}$ and varying $i^{2}$, we obtain

$$
i^{2}=\frac{N}{p} \frac{\psi^{2}\left(\alpha_{1} \theta+\alpha_{3}+\frac{\alpha_{5}}{\theta}\right)\left(\beta_{1} \theta+\beta_{3}+\frac{\beta_{5}}{\theta}\right)}{\psi\left(\beta_{1} \theta+\beta_{3}+\frac{\beta_{5}}{\theta}\right)-\frac{N}{p}} .
$$


Minimizing this expression (3.3), i.e. $\frac{\partial i^{2}}{\partial \psi}=0, \frac{\partial i^{2}}{\partial \theta}=0$, gives us

$$
\begin{gathered}
\psi=\frac{2 N}{p\left(\beta_{1} \theta+\beta_{3}+\frac{\beta_{5}}{\theta}\right)}, \\
\left(\alpha_{1} \beta_{3}-\alpha_{3} \beta_{1}\right) \theta^{2}+2\left(\alpha_{1} \beta_{5}-\alpha_{5} \beta_{1}\right) \theta+\alpha_{3} \beta_{5}-\alpha_{5} \beta_{3}=0 .
\end{gathered}
$$

Substituting this value into (3.3), we get,

$$
i^{2}=\frac{4 N^{2}}{p^{2}} \frac{\left(\alpha_{1} \theta+\alpha_{3}+\frac{\alpha_{5}}{\theta}\right)}{\beta_{1} \theta+\beta_{3}+\frac{\beta_{5}}{\theta}} .
$$

By taking into account (3.2), from this expression, we obtain

$$
\begin{aligned}
i^{2}=\frac{4 N^{2}}{p^{2}} & \left\{\left(\frac{1}{4 C}+\frac{3 \phi^{\prime}}{4 N C}+\frac{\phi^{\prime}}{9 K C}\right) \theta^{2}+\left(1+\frac{1}{C}-\frac{2 \phi^{\prime}}{9 K C}\right) \theta+\frac{1}{C}+\frac{\phi^{\prime}}{9 K C}\right\} \\
\cdot & \left\{\left(\frac{3}{4}+\frac{N}{4 \phi^{\prime}}+\frac{N}{9 K}\right) \theta^{2}+\left(3-\frac{N}{\phi^{\prime}}+\frac{2 N}{9 K}\right) \theta+\frac{N}{\phi^{\prime}}+\frac{N}{9 K}\right\}^{-1}
\end{aligned}
$$

where $\theta$ is a solution of the second equation of (3.4).

For the long cylindrical shell i.e., $\psi=1, \theta \ll 1$, we deduce from (3.3)

$$
i^{2}=\frac{N \alpha_{5} \beta_{5}}{p\left(\beta_{5} \theta-\frac{N}{p} \theta^{2}\right)}
$$

The minimization of this expression $i^{2}$ yields

$$
\frac{\partial i^{2}}{\partial \theta}=-\frac{N \alpha_{5} \beta_{5}\left(p \beta_{5}-2 N \theta\right)}{\left(p \beta_{5} \theta-N \theta^{2}\right)^{2}}=0
$$

from here, we obtain $\theta=\frac{p \beta_{5}}{2 N}=\theta_{*}$.

Substituting this value into $\frac{\partial^{2} i^{2}}{\partial \theta^{2}}$, we have the sufficient condition of minimum

$$
\left.\frac{\partial^{2} i^{2}}{\partial \theta^{2}}\right|_{\theta=\theta_{*}}=\frac{32 N^{4}}{p^{4}} \frac{\alpha_{5}}{\beta_{5}^{3}}=\frac{32 N^{4}}{p^{4}} \frac{\frac{1}{C}+\frac{\phi^{\prime}}{9 K C}}{\left(\frac{N}{\phi^{\prime}}+\frac{N}{9 K}\right)^{3}} .
$$


Since $C=1+\frac{3 N+\phi^{\prime}}{9 K}>0$, then $\left.\frac{\partial^{2} i^{2}}{\partial \theta^{2}}\right|_{\hat{\theta}=\theta_{*}}>0$. Finally, we receive the expression for finding the critical loads as follows

\section{Remarks}

$$
i^{2}=\frac{4 N^{2}}{p^{2}}\left(\frac{\frac{1}{C}+\frac{\phi^{\prime}}{9 K C}}{\frac{N}{\phi^{\prime}}+\frac{N}{9 K}}\right) .
$$

* If the material is incompressible i.e. $K \rightarrow+\infty, C=1$, then (3.5) and (3.6) return to the results in $[1,4,5]$.

* The obtained result (3.5) coincides with the one given in [3].

\subsection{Cylindrical shell subjected to external pressure}

The pre-buckling process is of the form

$$
\begin{aligned}
& \sigma_{22}=-\frac{R}{h} \tilde{q} \equiv-q, \quad \sigma_{11}=\sigma_{12}=0, \quad \sigma_{u}=\left|\sigma_{22}\right|=q \\
& \dot{\varepsilon}_{11}=\frac{\dot{q}}{2 \phi^{\prime}}-\frac{\dot{q}}{9 K}, \quad \dot{\varepsilon}_{22}=-\frac{\dot{q}}{\phi^{\prime}}-\frac{\dot{q}}{9 K}, \quad \dot{\varepsilon}_{33}=\frac{\dot{q}}{2 \phi^{\prime}}-\frac{\dot{q}}{9 K}, \quad \dot{\varepsilon}_{12}=0, \\
& \frac{d s}{d t}=\frac{\dot{q}(t)}{\phi^{\prime}(s)} \quad \text { or } \quad s=\phi^{-1}\left(\sigma_{u}\right)=\phi^{-1}(q) .
\end{aligned}
$$

We can determine, from (1.6) and (1.7), the coefficients $\alpha_{i}, \beta_{i}$ as follows

$$
\begin{array}{lll}
\alpha_{1}=\frac{1}{C}+\frac{\phi^{\prime}}{9 K C}, & \alpha_{2}=\alpha_{4}=0, \\
\alpha_{3}=1+\frac{1}{C}-\frac{2 \phi^{\prime}}{9 K C}, & \alpha_{5}=\frac{1}{4 C}+\frac{3 \phi^{\prime}}{4 N C}+\frac{\phi^{\prime}}{9 K C}, \\
\beta_{1}=\frac{N}{\phi^{\prime}}+\frac{N}{9 K}, & \beta_{2}=\beta_{4}=0, \\
\beta_{3}=3-\frac{N}{\phi^{\prime}}+\frac{2 N}{9 K}, & \beta_{5}=\frac{3}{4}+\frac{N}{4 \phi^{\prime}}+\frac{N}{9 K}, \\
C=1+\frac{3 N+\phi^{\prime}}{9 K} . &
\end{array}
$$

In this case, the expression (2.6) is given by

$$
i^{2}=\frac{N \psi^{2}\left(\alpha_{1} \beta^{4}+\alpha_{3} \beta^{2}+\alpha_{5}\right)\left(\beta_{1} \beta^{4}+\beta_{3} \beta^{2}+\beta_{5}\right)}{q\left(\beta_{1} \beta^{4}+\beta_{3} \beta^{2}+\beta_{5}\right) \psi-N \beta^{4}} .
$$

By denoting $\theta=\beta^{2}=\left(\frac{m \pi R}{n L}\right)^{2}$, we can rewrite the above equation in the form

$$
i^{2}=\frac{N \psi^{2}\left(\alpha_{1} \theta+\alpha_{3}+\frac{\alpha_{5}}{\theta}\right)\left(\beta_{1} \theta+\beta_{3}+\frac{\beta_{5}}{\theta}\right)}{\frac{q}{\theta}\left(\beta_{1} \theta+\beta_{3}+\frac{\beta_{5}}{\theta}\right) \psi-N} .
$$


Minimizing this relation i.e. $\frac{\partial i^{2}}{\partial \psi}=0, \frac{\partial i^{2}}{\partial \theta}=0$, we get, an expression for finding critical load

$$
\begin{gathered}
i^{2}=\frac{4 N^{2}}{q^{2}} \frac{\theta^{2}\left(\alpha_{1} \theta+\alpha_{3}+\frac{\alpha_{5}}{\theta}\right)}{\left(\beta_{1} \theta+\beta_{3}+\frac{\beta_{5}}{\theta}\right)}, \\
\left(\alpha_{1}-\frac{\alpha_{5}}{\theta^{2}}\right)\left(\beta_{1} \theta+\beta_{3}+\frac{\beta_{5}}{\theta}\right)-\left(\beta_{1}-\frac{\beta_{5}}{\theta^{2}}\right)\left(\alpha_{1} \theta+\alpha_{3}+\frac{\alpha_{5}}{\theta}\right) \\
+\frac{2}{\theta}\left(\alpha_{1} \theta+\alpha_{3}+\frac{\alpha_{5}}{\theta}\right)\left(\beta_{1} \theta+\beta_{3}+\frac{\beta_{5}}{\theta}\right)=0 .
\end{gathered}
$$

Substituting $\alpha_{i}, \beta_{i}(i=1,2,3)$ into the first relation of (3.10), we obtain

$$
\begin{aligned}
& i^{2}=\frac{4 N^{2}}{q^{2}} \theta^{2}\left\{\left(\frac{1}{C}+\frac{\phi^{\prime}}{9 K C}\right) \theta^{2}+\left(1+\frac{1}{C}-\frac{2 \phi^{\prime}}{9 K C}\right) \theta+\frac{1}{4 C}+\frac{3 \phi^{\prime}}{4 N C}\right. \\
& \left.+\frac{\phi^{\prime}}{9 K C}\right\} \cdot\left\{\left(\frac{N}{\phi^{\prime}}+\frac{N}{9 K}\right) \theta^{2}+\left(3-\frac{N}{\phi^{\prime}}+\frac{2 N}{9 K}\right) \theta+\frac{3}{4}+\frac{N}{4 \phi^{\prime}}+\frac{N}{9 K}\right\}^{-1},
\end{aligned}
$$

where $\theta$ is a solution of the second equation of (3.10).

For long cylindrical shells i.e. $\psi=1, \theta \ll 1$, the expression (3.9) gives us

$$
i^{2}=\frac{N}{q}\left(\alpha_{5}+\frac{i^{2} \theta^{2}}{\psi \beta_{5}}\right) \quad \text { or } \quad i^{2}=\frac{N \alpha_{5} \beta_{5}}{q \beta_{5}-N \theta^{2}} .
$$

By the same method, we get $\frac{\partial \dot{i}^{2}}{\partial \theta}=0$ i.e. $\theta_{*}=0$; the sufficient condition of minimum is satisfied:

$$
\left.\frac{\partial^{2} i^{2}}{\partial \theta^{2}}\right|_{\theta=\theta_{*}}=\frac{2 N^{2}}{q^{2}} \frac{\alpha_{5}}{\beta_{5}}=\frac{2 N^{2}}{q^{2}} \frac{\frac{1}{4 C}+\frac{3 \phi^{\prime}}{4 N C}+\frac{\phi^{\prime}}{9 K C}}{\frac{3}{4}+\frac{N}{4 \phi^{\prime}}+\frac{N}{9 K}}>0 .
$$

So the minimal value of $i^{2}$ leads to

$$
i^{2}=\frac{N}{q} \alpha_{5}=\frac{N}{q}\left(\frac{1}{4 C}+\frac{3 \phi^{\prime}}{4 N C}+\frac{\phi^{\prime}}{9 K C}\right)
$$

where $C=1+\frac{3 N+\phi^{\prime}}{9 K}>0$.

\section{Remarks}

* If a shell material is incompressible, then (3.11) and (3.12) coincide with the results in $[1,4,5]$.

* According to (3.7) $N, \phi^{\prime}(s)$ can be represented as functions of $q$, from (3.12) we can determine critical $q^{*}$. 


\subsection{Cylindrical shell subjected to torsion at its butt-ends}

Let, us consider a cylindrical shell of radius $R$, length $L$ and thickness $h$ subjected to torsion by a pair of moments $M_{k}=2 \pi h R^{2} \tau$ at the two butt-ends. Suppose that a material of shell is compressible and a kinematic boundary condition is simply supported at the planes $x_{1}=0$ and $x_{1}=L$.

The pre-buckling process, in this case, is represented as

$$
\begin{aligned}
& \sigma_{12}=-\tau, \quad \sigma_{11}=\sigma_{22}=0, \quad \sigma_{u}=\sqrt{3}\left|\sigma_{12}\right|, \\
& \dot{\varepsilon}_{11}=\dot{\varepsilon}_{22}=\dot{\varepsilon}_{33}=0, \quad \dot{\varepsilon}_{12}=-\frac{3 \dot{\tau}}{2 \phi^{\prime}}, \\
& \frac{d s}{d t}=\frac{\sqrt{3} \dot{\tau}}{\phi^{\prime}(s)} \quad \text { or } \quad s=\phi^{-1}\left(\sigma_{u}\right)=\phi^{-1}(\sqrt{3} \tau) .
\end{aligned}
$$

The coefficients $\alpha_{i}, \beta_{i}$ are calculated by the formulae

$$
\begin{aligned}
& \alpha_{1}=\alpha_{5}=\frac{1}{C}+\frac{N}{9 K C}, \alpha_{2}=\alpha_{4}=0, \alpha_{3}=\frac{1}{C}+\frac{\phi^{\prime}}{N}-\frac{2 N}{9 K C}, \\
& \beta_{1}=\beta_{5}=1+\frac{N}{9 K}, \beta_{2}=\beta_{4}=0, \beta_{3}=\frac{2 N}{9 K}+\frac{3 N}{\phi^{\prime}}-1, \\
& C=1+\frac{4 N}{9 K} .
\end{aligned}
$$

Putt.ing $i=\frac{3 R}{h}, \psi=n^{2}, \beta=\frac{m \pi R}{n L}$, the relation (2.6) takes the form

$$
i^{2}=\frac{N}{2 \tau}\left[\left(\alpha_{1} \beta^{3}+\alpha_{3} \beta+\frac{\alpha_{5}}{\beta}\right) \psi+\frac{i^{2} \beta^{3}}{\psi\left(\beta_{1} \beta^{4}+\beta_{3} \beta+\beta_{5}\right)}\right] .
$$

From here, one deduces

$$
i^{2}=\frac{N}{2 \tau} \frac{\left(\alpha_{1} \beta^{3}+\alpha_{3} \beta+\frac{\alpha_{5}}{\beta}\right)\left(\beta_{1} \beta^{4}+\beta_{3} \beta+\beta_{5}\right) \psi^{2}}{\psi\left(\beta_{1} \beta^{4}+\beta_{3} \beta+\beta_{5}\right)-\frac{N}{2 \tau} \beta^{3}} .
$$

Minimizing this expression, after series of calculations, gives us

$$
i^{2}=\frac{N^{2}}{\tau^{2}} \frac{\left(\alpha_{1} \beta^{6}+\alpha_{3} \beta^{4}+\alpha_{5} \beta^{2}\right)}{\left(\beta_{1} \beta^{4}+\beta_{3} \beta+\beta_{5}\right)},
$$

where $\beta$ is a solution of the following equation:

$$
\begin{aligned}
\left(3 \alpha_{1} \beta^{2}\right. & \left.+\alpha_{3}-\frac{\alpha_{5}}{\beta^{2}}\right)\left(\beta_{1} \beta^{4}+\beta_{3} \beta+\beta_{5}\right) \beta-\left(4 \beta_{1} \beta^{3}+\beta_{3}\right)\left(\alpha_{1} \beta^{3}+\alpha_{3} \beta+\frac{\alpha_{5}}{\beta}\right) \beta \\
& +3\left(\alpha_{1} \beta^{3}+\alpha_{3} \beta+\frac{\alpha_{5}}{\beta}\right)\left(\beta_{1} \beta^{4}+\beta_{3} \beta+\beta_{5}\right)=0 .
\end{aligned}
$$


Substituting $\alpha_{i}, \beta_{i}(i=1,2,3)$ from (3.14) into (3.16), we obtain

$$
\begin{aligned}
i^{2}= & \frac{N^{2}}{\tau^{2}}\left[\left(\frac{1}{C}+\frac{N}{9 K C}\right) \beta^{6}+\left(\frac{1}{C}+\frac{\phi^{\prime}}{N}-\frac{2 N}{9 K C}\right) \beta^{4}+\left(\frac{1}{C}+\frac{N}{9 K C}\right) \beta^{2}\right] . \\
& \cdot\left[\left(1+\frac{N}{9 K}\right) \beta^{4}+\left(\frac{2 N}{9 K}+\frac{3 N}{\phi^{\prime}}-1\right) \beta+1+\frac{N}{9 K}\right]^{-1} .
\end{aligned}
$$

The relations (3.16), (3.17) let us define the critical loads.

For long cylindrical shells, i.e. $\psi=1, \beta^{2} \ll 1$, we receive from (3.15)

$$
i^{2}=\frac{N}{2 \tau} \frac{\alpha_{5} \beta_{5}}{\beta \beta_{5}-\frac{N}{2 \tau} \beta^{4}} .
$$

The minimization of $i^{2}$, i.e. $\frac{\partial i^{2}}{\partial \beta}=0$, yields

$$
\beta=\sqrt[3]{\frac{\tau \beta_{5}}{2 N}}=\beta_{*}
$$

Consider the sufficient condition of extremum

$$
\left.\frac{\partial^{2} i^{2}}{\partial \beta^{2}}\right|_{\beta=\beta_{\bullet}}=\frac{16}{3} \frac{N^{2}}{\tau^{2}} \frac{\alpha_{5}}{\beta_{5}}=\frac{16}{3} \frac{N^{2}}{\tau^{2}} \cdot \frac{1}{C} .
$$

Since $C=1+\frac{4 N}{9 K}>0$, then $\left.\frac{\partial^{2} i^{2}}{\partial \beta^{2}}\right|_{\beta=\beta_{*}}>0$, this condition is satisfied.

Substituting $\beta_{*}$ into the expression $i^{2}$, we have

$$
i^{2}=\left(\frac{2 N}{\tau}\right)^{4 / 3} \frac{\left(1+\frac{N}{9 K}\right)^{2 / 3}}{1+\frac{4 N}{9 K}}
$$

It is clear that the result (3.17) returns to the one in $[2,4]$, if the material is incompressible.

3.4. Cylindrical shell simultaneously subjected to compression along the generatrix and external pressure

Assume that the structure is subjected to compression $p$ and external pressure $q$. The material is compressible. In this case, the pre-buckling process is of the form

$$
\sigma_{11}=-p, \quad \sigma_{22}=-q, \quad \sigma_{12}=0, \quad \sigma_{u}^{2}=p^{2}-p q+q^{2} .
$$


Components of the strain velocity tensor determined according to the theory of elastoplastic processes [1] are given by the formulae [3]

$$
\begin{aligned}
& \dot{\varepsilon}_{11}=\frac{1}{N}\left(-\dot{p}+\frac{1}{2} \dot{q}\right)-Q(s, t)\left(p-\frac{1}{2} q\right)-\frac{\dot{p}+\dot{q}}{9 K}, \\
& \dot{\varepsilon}_{22}=\frac{1}{N}\left(-\dot{q}+\frac{1}{2} \dot{p}\right)-Q(s, t)\left(q-\frac{1}{2} p\right)-\frac{\dot{p}+\dot{q}}{9 K}, \\
& \dot{\varepsilon}_{33}=\frac{1}{2 N}(\dot{p}+\dot{q})+\frac{1}{2} Q(s, t)(p+q)-\frac{\dot{p}+\dot{q}}{9 K}, \\
& \dot{\varepsilon}_{12}=0, \\
& Q(s, t)=\frac{1}{\sigma_{u}^{2}}\left(\frac{1}{\phi^{\prime}}-\frac{1}{N}\right)\left(p \dot{p}+q \dot{q}-\frac{1}{2} p \dot{q}-\frac{1}{2} q \dot{p}\right), \\
& \phi^{\prime}=\phi^{\prime}(s), \quad N=\frac{\sigma_{u}}{s} .
\end{aligned}
$$

The coefficients $\alpha_{i}, \beta_{i}$ are calculated as follows

$$
\begin{aligned}
& \alpha_{1}=\frac{1}{C}-\frac{3}{4 C}\left(1-\frac{\phi^{\prime}}{N}\right) \frac{p^{2}}{\sigma_{u}^{2}}+\frac{\phi^{\prime}}{9 K C}, \quad \alpha_{2}=\alpha_{4}=0, \\
& \alpha_{3}=1+\frac{1}{C}-\frac{3}{2 C}\left(1-\frac{\phi^{\prime}}{N}\right) \frac{p q}{\sigma_{u}^{2}}-\frac{2 \phi^{\prime}}{9 K C}, \\
& \alpha_{5}=\frac{1}{C}-\frac{3}{4 C}\left(1-\frac{\phi^{\prime}}{N}\right) \frac{q^{2}}{\sigma_{u}^{2}}+\frac{\phi^{\prime}}{9 K C}, \\
& \beta_{1}=1+\frac{1}{4}\left(\frac{N}{\phi^{\prime}}-1\right)\left(\frac{2 q-p}{\sigma_{u}}\right)^{2}+\frac{N}{9 K}, \quad \beta_{2}=\beta_{4}=0, \\
& \beta_{3}=2\left(1+\frac{N}{9 K}\right)+\frac{1}{2}\left(\frac{N}{\phi^{\prime}}-1\right) \frac{(2 q-p)(2 p-q)}{\sigma_{u}^{2}}, \\
& \beta_{5}=1+\frac{1}{4}\left(\frac{N}{\phi^{\prime}}-1\right)\left(\frac{2 p-q}{\sigma_{u}}\right)^{2}+\frac{N}{9 K}, \\
& C=1+\frac{4 N}{9 K}+\frac{\left(\phi^{\prime}-N\right)}{9 K} \frac{(p+q)^{2}}{p^{2}-p q+q^{2}} .
\end{aligned}
$$

By the same method presented in the above part we obtain a relation for finding the critical load

$$
i^{2}=\frac{N}{p \beta^{2}+q}\left[\psi\left(\alpha_{1} \beta^{4}+\alpha_{3} \beta^{2}+\alpha_{5}\right)+\frac{i^{2} \beta^{4}}{\psi\left(\beta_{1} \beta^{4}+\beta_{3} \beta^{2}+\beta_{5}\right)}\right]
$$

or

$$
i^{2}=\frac{N \psi^{2}\left(\alpha_{1} \beta^{4}+\alpha_{3} \beta^{2}+\alpha_{5}\right)\left(\beta_{1} \beta^{4}+\beta_{3} \beta^{2}+\beta_{5}\right)}{\left(p \beta^{2}+q\right)\left(\beta_{1} \beta^{4}+\beta_{3} \beta^{2}+\beta_{5}\right) \psi-N \beta^{4}}
$$


where $i=\frac{3 R}{h}, \psi=n^{2}, \beta=\frac{m \pi R}{n L}$.

For calculating usefully, we represent $i^{2}$ in the another form by denoting $\theta=\beta^{2}$.

$$
i^{2}=\frac{N \psi^{2}\left(\alpha_{1} \theta+\alpha_{3}+\frac{\alpha_{5}}{\theta}\right)\left(\beta_{1} \theta+\beta_{3}+\frac{\beta_{5}}{\theta}\right)}{\left(p+\frac{q}{\theta}\right)\left(\beta_{1} \theta+\beta_{3}+\frac{\beta_{5}}{\theta}\right) \psi-N} .
$$

Minimizing this expression i.e. $\frac{\partial i^{2}}{\partial \psi}=0, \frac{\partial i^{2}}{\partial \theta}=0$, after some calculations, gives us

$$
\begin{gathered}
\psi=\frac{2 N}{\left(p+\frac{q}{\theta}\right)\left(\beta_{1} \theta+\beta_{3}+\frac{\beta_{5}}{\theta}\right)} \\
\left(\alpha_{1}-\frac{\alpha_{5}}{\theta^{2}}\right)\left(\beta_{1} \theta+\beta_{3}+\frac{\beta_{5}}{\theta}\right)-\left(\beta_{1}-\frac{\beta_{5}}{\theta^{2}}\right)\left(\alpha_{1} \theta+\alpha_{3}+\frac{\alpha_{5}}{\theta}\right) \\
+\frac{2 q}{\theta^{2}\left(p+\frac{q}{\theta}\right)}\left(\alpha_{1} \theta+\alpha_{3}+\frac{\alpha_{5}}{\theta}\right)\left(\beta_{1} \theta+\beta_{3}+\frac{\beta_{5}}{\theta}\right)=0 .
\end{gathered}
$$

So, substituting (3.22) into (3.21), we get

$$
i^{2}=\frac{4 N^{2}}{\left(p+\frac{q}{\theta}\right)^{2}}\left(\frac{\alpha_{1} \theta+\alpha_{3}+\frac{\alpha_{5}}{\theta}}{\beta_{1} \theta+\beta_{3}+\frac{\beta_{5}}{\theta}}\right) .
$$

By taking into account (3.20), from (3.24), we obtain

$$
\begin{aligned}
i^{2}= & \frac{4 N^{2} \theta^{2}}{(p \theta+q)^{2}}\left\{\left[\frac{1}{C}-\frac{3}{4 C}\left(1-\frac{\phi^{\prime}}{N}\right) \frac{p^{2}}{p^{2}-p q+q^{2}}+\frac{\phi^{\prime}}{9 K C}\right] \theta^{2}\right. \\
& +\left[1-\frac{2 \phi^{\prime}}{9 K C}+\frac{1}{C}-\frac{3}{2 C}\left(1-\frac{\phi^{\prime}}{N}\right) \frac{p q}{p^{2}-p q+q^{2}}\right] \theta+\frac{1}{C} \\
& \left.-\frac{3}{4 C}\left(1-\frac{\phi^{\prime}}{N}\right) \frac{q^{2}}{p^{2}-p q+q^{2}}+\frac{\phi^{\prime}}{9 K C}\right\} \cdot\left\{\left[1+\frac{1}{4}\left(\frac{N}{\phi^{\prime}}-1\right) \frac{(2 q-p)^{2}}{p^{2}-p q+q^{2}}\right.\right. \\
& \left.+\frac{N}{9 K}\right] \theta^{2}+\left[2\left(1+\frac{N}{9 K}\right)+\frac{1}{2}\left(\frac{N}{\phi^{\prime}}-1\right) \frac{(2 q-p)(2 p-q)}{p^{2}-p q+q^{2}}\right] \theta \\
& \left.+1+\frac{1}{4}\left(\frac{N}{\phi^{\prime}}-1\right) \frac{(2 p-q)^{2}}{p^{2}-p q+q^{2}}+\frac{N}{9 K}\right\}^{-1} .
\end{aligned}
$$

where $\theta$ is a solution of the equation of (3.23). 
Applying the loading parameter method [1] we solve simultaneously the equitions (1.3) and (3.25). After finding the critical value $t_{*}$, we can determine the critical loads as follows

$$
p^{*}=p\left(t_{*}\right), \quad q^{*}=q\left(t_{*}\right) .
$$

For long cylindrical shells, see $[1,2]$, we get

$$
\psi=1, \quad \theta \ll 1, \quad i^{2}=\frac{N \alpha_{5} \beta_{5}}{(p \theta+q) \beta_{5}-N \theta^{2}} .
$$

Minimizing this expression i.e. $\frac{\partial i^{2}}{\partial \theta}=0$, gives us

$$
\theta=\frac{p \beta_{5}}{2 N} \equiv \theta_{*}
$$

Now consider the sufficient condition of extremum

$$
\left.\frac{\partial^{2} i^{2}}{\partial \theta^{2}}\right|_{\theta=\theta_{*}}=\frac{2 \alpha_{5} \beta_{5} N^{2}}{\left(\frac{p^{2} \beta_{5}^{2}}{4 N}+q \beta_{5}\right)^{2}} .
$$

We will demonstrate $\alpha_{5}>0, \beta_{5}>0$. In reality, we have

$$
\begin{aligned}
C & =1+\frac{4 N}{9 K}+\frac{\phi^{\prime}-N}{9 K} \frac{(p+q)^{2}}{\sigma_{u}^{2}} \\
& =1+\frac{\phi^{\prime}}{9 K} \frac{(p+q)^{2}}{\sigma_{u}^{2}}+\frac{N}{3 K} \frac{(p-q)^{2}}{\sigma_{u}^{2}}>0 \\
\alpha_{5} & =\frac{1}{C}\left[1-\frac{3}{4}\left(1-\frac{\phi^{\prime}}{N}\right) \frac{q^{2}}{\sigma_{u}^{2}}+\frac{\phi^{\prime}}{9 K}\right] \\
& =\frac{1}{C}\left[\frac{(2 p-q)^{2}}{4 \sigma_{u}^{2}}+\frac{3}{4} \frac{\phi^{\prime} q^{2}}{N \sigma_{u}^{2}}+\frac{\phi^{\prime}}{9 K}\right]>0 \\
\beta_{5} & =1+\frac{1}{4}\left(\frac{N}{\phi^{\prime}}-1\right) \frac{(2 p-q)^{2}}{\sigma_{u}^{2}}+\frac{N}{9 K} \\
& =\frac{3 q^{2}}{4 \sigma_{u}^{2}}+\frac{N}{4 \phi^{\prime}} \frac{(2 p-q)^{2}}{\sigma_{u}^{2}}+\frac{N}{9 K}>0
\end{aligned}
$$

So $\left.\frac{\partial^{2} i^{2}}{\partial \theta^{2}}\right|_{\theta=\theta_{*}}>0$, the sufficient condition of minimum is satisfied.

Substituting $\alpha_{5}, \beta_{5}$ from (3.20) into (3.26), this relation takes the form

$$
i^{2}=\frac{4 N^{2}\left[\frac{1}{C}-\frac{3}{4 C}\left(1-\frac{\phi^{\prime}}{N}\right) \frac{q^{2}}{p^{2}-p q+q^{2}}+\frac{\phi^{\prime}}{9 K C}\right]}{p^{2}\left[1+\frac{1}{4}\left(\frac{N}{\phi^{\prime}}-1\right) \frac{(2 p-q)^{2}}{p^{2}-p q+q^{2}}+\frac{N}{9 K}\right]+4 N q} .
$$


Expressing $p=p(t), q=q(t)$ one can find the critical value of loading parameter $t_{*}$ from (1.3) and (3.27). The critical loads will be given $p^{*}=p\left(t_{*}\right), q^{*}=q\left(t_{*}\right)$.

\section{Remarks}

* If a shell material is incompressible, then (3.25) and (3.27) coincide with the results obtained in $[1,4]$.

* If the compression $p$ is equal to zero, then (3.25) returns to (3.5).

*. If the pressure $q$ takes the value equal to zero, then we can obtain from (3.25) the result (3.11).

\section{Numerical examples and discussion}

Let us consider a long cylindrical shell subjected to compression $p$ along the generatrix and external pressure $q$ which depend on a loading parameter $t$ as follows

$$
p(t)=\frac{\left(q_{0}+q_{1} t\right)^{2}}{q_{2}} ; \quad q(t)=q_{0}+q_{1} t
$$

where $q_{0}, q_{1}, q_{2}$ are known constants.

Substituting (4.1) into (3.27), we obtain

$$
\begin{aligned}
& i \equiv \frac{3 R}{h}=\frac{2 q_{2} N}{\left(q_{0}+q_{1} t\right)^{2}}\left\{\frac{1}{C}-\frac{3}{4 C}\left(1-\frac{\phi^{\prime}}{N}\right) \frac{\left(q_{0}+q_{1} t\right)^{2}}{\sigma_{u}^{2}}+\frac{\phi^{\prime}}{9 K C}\right\}^{\frac{1}{2}} \cdot \\
& \cdot\left\{1+\frac{1}{4}\left(\frac{N}{\phi^{\prime}}-1\right)\left[\frac{2\left(q_{0}+q_{1} t\right)^{2}}{q_{2}}-\left(q_{0}+q_{1} t\right)\right]^{2} \cdot \frac{1}{\sigma_{u}^{2}}+\frac{N}{9 K}+\frac{4 N q_{2}^{2}}{\left(q_{0}+q_{1} t\right)^{3}}\right\}^{-\frac{1}{2}}
\end{aligned}
$$

where

$$
\sigma_{u}=\sigma_{u}(t)=\left[\frac{\left(q_{0}+q_{1} t\right)^{4}}{q_{2}^{2}}-\frac{\left(q_{0}+q_{1} t\right)^{3}}{q_{2}}+\left(q_{0}+q_{1} t\right)^{2}\right]^{\frac{1}{2}} ; \phi_{\iota}^{\prime}=\phi^{\prime}(s) ; N=\frac{\sigma_{u}}{s} .
$$

If the shell is incompressible, we have $K \rightarrow+\infty, C=1$ and

$$
\begin{aligned}
i & \equiv \frac{3 R}{h}=\frac{2 q_{2} N}{\left(q_{0}+q_{1} t\right)^{2}}\left\{1-\frac{3}{4}\left(1-\frac{\phi^{\prime}}{N}\right) \frac{\left(q_{0}+q_{1} t\right)^{2}}{\sigma_{u}^{2}}\right\}^{\frac{1}{2}} \\
& \cdot\left\{1+\frac{1}{4}\left(\frac{N}{\phi^{\prime}}-1\right)\left[\frac{2\left(q_{0}+q_{1} t\right)^{2}}{q_{2}}-\left(q_{0}+q_{1} t\right)\right]^{2} \cdot \frac{1}{\sigma_{u}^{2}}+\frac{4 N q_{2}^{2}}{\left(q_{0}+q_{1} t\right)^{3}}\right\}^{-\frac{1}{2}} .
\end{aligned}
$$

The arc-length of stain trajectory in (1.3) is calculated by the Euler iterative method

$$
s\left(t_{n+1}\right)=s\left(t_{n}\right)+\tau F\left(t_{n}, s\left(t_{n}\right)\right) ; \quad \tau=t_{n+1}-t_{n} .
$$

In order to investigate the influence of compressibility of material on the stability of cylindrical shell, we will calculate and compare the critical forces of the compressible shell with the ones of incompressible shell. 
A numerical analysis is considered on the long cylindrical shell made of steel $30 \mathrm{XCA}$ with an elastic modulus $3 G=2.6 \cdot 10^{5} \mathrm{MPa}$, an yield point $\sigma_{s}=400 \mathrm{MPa}$, the constants $q_{0}=2 \mathrm{MPa} ; q_{1}=0.1 \mathrm{MPa} ; q_{2}=0.1 \mathrm{MPa}$, the iterative step $\tau=0.01$. The ratio $\frac{R}{h}$ varies from 22 to 49 and from 50 to 77 with the arithmetical ratio equal to 3 . The coefficients $K$ and $\nu$ are taken the value

$$
K=\frac{E}{3(1-2 \nu)} ; \quad E=2 G(1+\nu)
$$

$$
\nu=0.310 ; 0.320 ; 0.330 ; 0.350 ; 0.400 ; 0.410 ; 0.420 ; 0.430 ; 0.440
$$

Hereafter, we give the numerical results in two cases

1. The Poisson coefficient $\nu$ varies, while the ratio $\frac{R}{h}$ is constant (see table 1 )

2. The slenderness of shell $i=\frac{3 R}{h}$ varies, while the Poisson coefficient $\nu$ is constant (see table 2)

\begin{tabular}{|c|c|c|c|c|c|}
\hline$\nu$ & $t^{*}$ & $s \cdot 10^{3}$ & $p^{*} \mathrm{MPa}$ & $q^{*} \mathrm{MPa}$ & $\sigma_{u}^{*} \mathrm{MPa}$ \\
\hline \multicolumn{6}{|c|}{ Ratio $R / h=22 ; i=66$} \\
\hline 0.500 & 56.61 & 9.0148 & 586.8 & 7.66 & 583.0 \\
\hline 0.310 & 51.92 & 7.8972 & 517.1 & 7.19 & 513.5 \\
\hline 0.320 & 52.11 & 7.9699 & 519.8 & 7.21 & 516.3 \\
\hline 0.330 & 52.30 & 8.0007 & 522.6 & 7.23 & 519.0 \\
\hline 0.340 & 52.48 & 8.0252 & 525.2 & 7.25 & 521.6 \\
\hline 0.350 & 52.69 & 8.0871 & 528.2 & 7.27 & 524.6 \\
\hline 0.400 & 53.87 & 8.4108 & 545.5 & 7.39 & 541.9 \\
\hline 0.410 & 54.11 & 8.4718 & 549.1 & 7.41 & 545.4 \\
\hline 0.420 & 54.36 & 8.5386 & 552.8 & 7.44 & 549.1 \\
\hline 0.430 & 54.63 & 8.5904 & 556.8 & 7.46 & 553.1 \\
\hline 0.440 & 54.90 & 8.6479 & 560.9 & 7.49 & 557.1 \\
\hline \multicolumn{6}{|c|}{ Ratio $R / h=40 ; i=120$} \\
\hline 0.500 & 51.14 & 3.4970 & 505.9 & 7.11 & 502.4 \\
\hline 0.310 & 46.57 & 2.7527 & 443.0 & 6.66 & 439.7 \\
\hline 0.320 & 46.73 & 2.7857 & 445.2 & 6.67 & 441.9 \\
\hline 0.330 & 46.92 & 2.8154 & 447.7 & 6.69 & 444.4 \\
\hline 0.340 & 47.12 & 2.8550 & 450.4 & 6.71 & 447.1 \\
\hline 0.350 & 47.29 & 2.8756 & 452.7 & 6.73 & 449.3 \\
\hline 0.400 & $48: 38$ & 3.0512 & 467.4 & 6.84 & 464.1 \\
\hline 0.410 & 48.62 & 3.0968 & 470.7 & 6.86 & 467.3 \\
\hline 0.420 & 48.86 & 3.1383 & 474.0 & 6.89 & 470.6 \\
\hline
\end{tabular}

Table 1 


$\begin{array}{llllll}0.430 & 49.14 & 3.1838 & 477.9 & 6.91 & 474.5 \\ 0.440 & 49.42 & 3.2317 & 481.8 & 6.94 & 478.3\end{array}$

Ratio $R / h=62 ; i=186$

$\begin{array}{llllll}0.500 & 46.54 & 1.7579 & 442.6 & 6.65 & 439.3 \\ 0.310 & 28.41 & 1.0212 & 234.3 & 4.84 & 231.9 \\ 0.320 & 29.34 & 1.0530 & 243.3 & 4.93 & 240.9 \\ 0.330 & 30.29 & 1.0859 & 252.8 & 5.03 & 250.3 \\ 0.340 & 31.27 & 1.1204 & 262.8 & 5.13 & 260.2 \\ 0.350 & 32.28 & 1.1566 & 273.2 & 5.23 & 270.6 \\ 0.400 & 37.85 & 1.3669 & 334.5 & 5.78 & 331.7 \\ 0.410 & 39.08 & 1.4158 & 348.9 & 5.91 & 346.0 \\ 0.420 & 40.36 & 1.4676 & 364.2 & 6.04 & 361.2 \\ 0.430 & 41.68 & 1.5221 & 380.3 & 6.17 & 377.3 \\ 0.440 & 43.05 & 1.5797 & 397.4 & 6.30 & 394.3\end{array}$

Ratio $R / h=77 ; i=231$

\begin{tabular}{rrrrrr}
0.500 & 28.22 & 0.8848 & 232.4 & 4.82 & 230.0 \\
0.310 & 11.71 & 0.4357 & 100.5 & 3.17 & 98.9 \\
0.320 & 12.33 & 0.4497 & 104.5 & 3.23 & 102.9 \\
0.330 & 12.97 & 0.4643 & 108.6 & 3.30 & 107.0 \\
0.340 & 13.63 & 0.4796 & 113.0 & 3.36 & 111.4 \\
0.350 & 14.31 & 0.4956 & 117.6 & 3.43 & 116.0 \\
0.400 & 18.08 & 0.5896 & 144.9 & 3.81 & 143.1 \\
0.410 & 18.92 & 0.6117 & 151.4 & 3.89 & 149.5 \\
0.420 & 19.79 & 0.6350 & 158.2 & 3.98 & 156.3 \\
0.430 & 20.70 & 0.6599 & 165.6 & 4.07 & 163.6 \\
0.440 & 21.64 & 0.6861 & 173.3 & 4.16 & 171.3 \\
\hline
\end{tabular}

Table 2

\begin{tabular}{|c|c|c|c|c|c|c|}
\hline$R / h$ & $i$ & $t^{*}$ & $s \cdot 10^{3}$ & $p^{*} \mathrm{MPa}$ & $q^{*} \mathrm{MPa}$ & $\sigma_{u}^{*} \mathrm{MPa}$ \\
\hline \multicolumn{7}{|c|}{$\nu=0.500$} \\
\hline 22 & 66 & 55.61 & 9.0148 & 586.8 & 7.66 & 583.0 \\
\hline 25 & 75 & 55.19 & 7.2518 & 565.2 & 7.52 & 561.5 \\
\hline 28 & 84 & 54.07 & 6.0078 & 548.5 & 7.41 & 544.8 \\
\hline 31 & 93 & 53.14 & 5.0610 & 534.8 & 7.31 & 531.2 \\
\hline 34 & 102 & 52.48 & 4.4502 & 525.2 & 7.25 & 521.6 \\
\hline 37 & 111 & 51.88 & 3.9910 & 516.5 & 7.19 & 513.0 \\
\hline 40 & 120 & 51.14 & 3.4970 & 505.9 & 7.11 & 502.4 \\
\hline
\end{tabular}




\begin{tabular}{rcrrrrr}
43 & 129 & 50.42 & 3.0470 & 495.8 & 7.04 & 492.3 \\
46 & 138 & 49.90 & 2.7508 & 488.5 & 6.99 & 485.0 \\
49 & 147 & 49.43 & 2.5295 & 481.9 & 6.94 & 478.5 \\
50 & 150 & 49.27 & 2.4634 & 479.7 & 6.93 & 476.3 \\
53 & 159 & 48.70 & 2.2608 & 471.8 & 6.87 & 468.4 \\
56 & 168 & 48.03 & 2.0577 & 462.7 & 6.80 & 459.3 \\
59 & 177 & 47.46 & 1.9178 & 455.0 & 6.75 & 451.6 \\
62 & 186 & 46.54 & 1.7579 & 442.6 & 6.65 & 439.3 \\
65 & 195 & 45.19 & 1.6356 & 424.8 & 6.52 & 421.6 \\
68 & 204 & 41.15 & 1.4261 & 373.8 & 6.11 & 370.8 \\
71 & 213 & 36.35 & 1.2102 & 317.4 & 5.63 & 314.6 \\
74 & 222 & 32.06 & 1.0321 & 270.9 & 5.21 & 268.4 \\
77 & 231 & 28.22 & 0.8848 & 232.4 & 4.82 & 230.0 \\
$\nu$ & 0.310 & & & & & \\
22 & 66 & 51.92 & 7.8972 & 517.1 & 7.19 & 513.5 \\
25 & 75 & 50.67 & 6.3290 & 499.3 & 7.07 & 495.8 \\
28 & 84 & 49.61 & 5.1344 & 484.4 & 6.96 & 481.0 \\
31 & 93 & 48.96 & 4.3813 & 474.0 & 6.89 & 470.6 \\
34 & 102 & 48.11 & 3.7855 & 463.8 & 6.81 & 460.4 \\
37 & 111 & 47.23 & 3.1718 & 451.9 & 6.72 & 448.5 \\
40 & 120 & 46.57 & 2.7527 & 443.0 & 6.66 & 439.7 \\
43 & 129 & 46.00 & 2.4685 & 435.5 & 6.60 & 432.2 \\
46 & 138 & 45.26 & 2.1900 & 425.8 & 6.53 & 422.5 \\
49 & 147 & 44.43 & 1.9405 & 415.0 & 6.44 & 411.8 \\
50 & 150 & 44.15 & 1.8786 & 411.4 & 6.41 & 408.2 \\
53 & 159 & 43.51 & 1.7620 & 403.2 & 6.35 & 400.1 \\
56 & 168 & 38.70 & 1.5042 & 344.5 & 5.87 & 341.6 \\
59 & 177 & 33.22 & 1.2354 & 283.1 & 5.32 & 280.5 \\
62 & 186 & 28.41 & 1.0212 & 234.3 & 4.84 & 231.9 \\
65 & 195 & 24.20 & 0.8504 & 195.3 & 4.42 & 193.1 \\
68 & 204 & 20.48 & 0.7126 & 163.8 & 4.05 & 161.8 \\
71 & 213 & 17.20 & 0.6011 & 138.3 & 3.72 & 136.5 \\
74 & 222 & 14.30 & 0.5104 & 117.6 & 3.43 & 115.9 \\
77 & 231 & 11.71 & 0.4357 & 100.5 & 3.17 & 99.9 \\
\hline & & & & & & \\
& & & &
\end{tabular}

From the above results we can lead some conclusions

1. Theory of elastoplastic processes can be applied to the stability problem of cylindrical shell when both pre-buckling and post-buckling processes are complicated

2. The compressibility of material has an influence on the stability of structure. The more the-Poisson coefficient $\nu$ decreases, the more the value of critical force 
diminishes when the ratio $\frac{R}{h}$ is constant. This remark is deduced from the results on the table 1.

3. The more the shell is thin i.e. the slenderness $i$ increases, the more influence of compressibility is great. This observation can be seen on the table 2 .

4. For long shells we have shown the necessary and sufficient conditions of extremum. In general case, the sufficient condition, by reason of mathematical complication, will be verified in numerical examples.

5. When a material is incompressible, the obtained results return to the previous well-known ones (see $[1,2,4,5)$

6. Associating with the loading parameter method, the proposed method gives a way to solve efficiently series of stability problems of cylindrical shells.

This paper is completed with financial support from the National Basic Research Program in Natural Sciences.

\section{REFERENCES}

1. Dao Huy Bich. Theory of elastoplastic processes. Vietnam National University Publishing House, Hanoi 1999 (in Vietnamese).

2. Volmir A. S. Stability of deformable systems. Moscow 1963 (in Russian).

3. Dao Van Dung. Stability of cylindrical shells with compressible material in the theory of elastoplastic processes. Proceedings of the Fifth National Conference on Solid Mechanics, Hanoi, November 29 $\div 30,1996$, pp. 152-159 (in Vietnamese).

4. Dao Van Dung. Stability problem outside elastic limit according to the theory of elastoplastic deformation processes. Ph. D. Thesis, Hanoi 1993 (in Vietnamese).

5. Dao Van Dung. Stability of thin shell subjected to complex loading. Journal of Mechanics, Vol. 10, No 1, 1988, pp. 8-16 (in Vietnamese).

Received August 18, 2000

PHƯONG PHÁP GIẢI BÀI TOÁN ỔN ĐỊNH ĐÀN DĖO CỦA VỎ TRỤ VỚI VẬT LIỆU NÉN ĐƯợC CHỊU QUÁ TRİNH ĐẶT TÀI PHỨC TẠP

Bài này nhằm nghiên cứu lời giải và phương pháp xác định tái tới hạn trong bài toán ổn định đàn dèo của kết cấu chịu quá trình đặt tải phức tạp. Các kết quá thu được cho thấy ảnh hương của tính nén được của vật liệu lên sự ồn dịnh của vó trụ. Khi vật liệu là không nén được, ta nhận được các kết quả đã có trước đây.

Vietnam National University

Faculty of Mathematics, Mechanics and Informatics

334 Nguyen Trai Street, Hanoi, Vietnam 